\title{
MicroRNA-652 inhibits the biological characteristics of esophageal squamous cell carcinoma by directly targeting fibroblast growth factor receptor 1
}

\author{
CHENG ZHEN, JINGSHAN HUANG and JIBIN LU
}

Department of Thoracic Surgery, Shengjing Hospital of China Medical University, Shenyang, Liaoning 110000, P.R. China

Received September 1, 2018; Accepted August 28, 2019

DOI: $10.3892 /$ etm.2019.8072

\begin{abstract}
Numerous studies have demonstrated that microRNAs (miRNAs) are dysregulated in esophageal squamous cell carcinoma (ESCC). Changes in miRNA expression may be associated with ESCC formation and progression. Therefore, the identification of ESCC-associated miRNAs may facilitate the development of effective therapeutic approaches for patients with ESCC. Recently, miRNA-652 (miR-652) was recognized as a cancer-associated miRNA in a number of different types of cancer. However, the expression status and roles of miR-652 in ESCC as well as the molecular mechanisms modulated or altered by it remain largely unknown. In the present study, it was demonstrated that miR-652 was downregulated in ESCC tissues and cell lines. Functional assays showed that upregulation of miR-652 expression decreased proliferation and invasion of ESCC cells. Mechanistically, fibroblast growth factor receptor 1 (FGFR1) was determined to be a direct target of miR-652 in ESCC cells. Additionally, FGFR1 was upregulated in ESCC tissues, and the expression of FGFR1 was inversely correlated with miR-652 expression. Furthermore, restoring FGFR1 expression abolished the suppressive effects of miR-652 overexpression on the proliferation and invasion of ESCC cells. These findings demonstrated that miR-652 inhibits the proliferation and invasion of ESCC cells by directly targeting FGFR1.
\end{abstract}

\section{Introduction}

Esophageal carcinoma is the eighth most common cancer worldwide, and the sixth leading cause of cancer-associated deaths, with an estimated 455,800 new cases and 400,200 mortalities per year globally $(1,2)$. Based on histopathological analysis, esophageal carcinoma is divided into two subtypes,

Correspondence to: Professor Jibin Lu, Department of Thoracic Surgery, Shengjing Hospital of China Medical University, 36 Sanhao Road, Shenyang, Liaoning 110000, P.R. China

E-mail: sj_jibinlu@163.com

Key words: esophageal squamous cell carcinoma, microRNA-652, proliferation, invasion, fibroblast growth factor receptor 1 esophageal squamous cell carcinoma (ESCC) and esophageal adenocarcinoma (3). ESCC, accounts for about $90 \%$ of esophageal cancer cases and is characterized by invasiveness, recurrence and ability to metastasize (4). Despite the advances in diagnostic and surgical approaches, the clinical outcomes of patients with ESCC remain unsatisfactory with a 5 year survival rate of $26.2-49.4 \%$ (5). Genetic and epigenetic alterations are closely associated with the progression of ESCC; however, the detailed mechanisms underlying the pathogenesis of ESCC remain to be determined (6,7). Therefore, a detailed investigation into the formation and progression of ESCC is required to advance the identification of novel therapeutic targets for the treatment of patients with ESCC.

MicroRNAs (miRNAs) are a group of single stranded, non-coding RNA molecules of 18-24 nucleotides in length (8). miRNAs are expressed in plants, animals and some viruses, and have been considered as critical regulators of gene expression (9). miRNAs negatively regulate gene expression through base-pairing with the complementary sites in the 3'-untranslated regions (3'-UTRs) of their target genes (10) resulting in inhibition of translation and/or degradation of mRNAs (11). To date, miRNA molecules have been reported to be aberrantly expressed in almost all types of cancer, including ESCC (12), lung (13), colorectal (14), gastric (15) and prostate (16) cancer. Dysregulated miRNAs are implicated in the tumorigenesis and tumor development of ESCC via the regulation of cell proliferation, cell cycle, apoptosis, metastasis, angiogenesis, and resistance to radiation and chemotherapeutic treatment (17-19). miRNAs may function as tumor suppressors or oncogenes depending on the specific roles of their target genes $(20,21)$. Therefore, miRNAs may serve as therapeutic targets for the treatment of patients with ESCC.

miR-652 has been recognized as a cancer-associated miRNA in endometrial cancer (22), non-small cell lung cancer (23), pediatric acute lymphoblastic leukemia (24) and pancreatic cancer (25). However, the expression status and roles of miR-652 in ESCC as well as the molecular mechanisms involved remain largely unknown. Therefore, in the current study, miR-652 expression in ESCC and the biological roles of miR-652 in the development of ESCC were investigated. In addition, the potential mechanisms underlying these functions were explored. The present study aimed to determine the role of miR-652 in ESCC. 


\section{Materials and methods}

Patients and specimens. The present study was approved by The Ethics Committee of Shengjing Hospital of China Medical University (Liaoning, China). Written informed consent was obtained from all patients recruited in this research. In total, 37 pairs of ESCC tissues and adjacent non-tumor tissues ( $2 \mathrm{~cm}$ away from tumor tissues) were collected from patients (25 males, 12 females; age range, 51-73 years) who underwent surgery at the Shengjing Hospital of China Medical University between February 2015 and September 2017. None of the patients had been treated for cancer prior to surgical resection. All tissues were obtained and stored in liquid nitrogen until required for RNA and protein extraction.

Cell culture. A normal human esophageal epithelial cell line (HET-1A) was purchased from American Type Culture Collection. In total, four human ESCC cell lines, including KYSE70, KYSE150, TE-1, and Eca109, were purchased from The Cell Bank of Type Culture Collection of the Chinese Academy of Sciences. All cell lines were grown in Dulbecco's modified Eagle's medium (DMEM) supplemented with $10 \%$ fetal bovine serum (FBS) and 1\% streptomycin/penicillin mixture (all from Invitrogen; Thermo Fisher Scientific, Inc.). Cells were maintained at $37^{\circ} \mathrm{C}$ in a humidified atmosphere containing $5 \% \mathrm{CO}_{2}$.

Cell transfection. miR-652 mimics (5'-AAUGGCGCCACU AGGGUUGUG-3') and miRNA mimic negative control (miR-NC; 5'-UUCUCCGAACGUGUCACGUTT-3') were synthesized by Shanghai GenePharma Co., Ltd. miR-652 mimics were used to increase the expression of miR-652, while miR-NC served as the negative control. Human FGFR1 overexpression vector lacking the 3'-UTR pCMV-FGFR1 and empty pCMV plasmid were constructed by the Chinese Academy of Sciences. Cells in the mid-log phase were collected and plated into six-well plates with a density of $5 \times 10^{5}$ cells/well. The aforementioned oligonucleotides (100 pmol) and vectors $(4 \mu \mathrm{g})$ were transiently transfected into cells using Lipofectamine ${ }^{\circledR}$ 2000 reagent (Invitrogen; Thermo Fisher Scientific, Inc.) according to the manufacturer's protocol and $48 \mathrm{~h}$ after transfection, reverse transcription-quantitative PCR (RT-qPCR) and transwell invasion assays were performed. Cell Counting Kit-8 (CCK-8) assay and western blot analysis were conducted at 24 and $72 \mathrm{~h}$ after transfection, respectively.

$R N A$ extraction and $R T-q P C R$. Total cellular RNA was extracted from tissue samples or cells using TRIzol ${ }^{\circledR}$ reagent (Invitrogen; Thermo Fisher Scientific, Inc.). For quantification of miR-652 expression, first-strand complementary DNA (cDNA) was prepared from total cellular RNA using a TaqMan MicroRNA Reverse Transcription kit using the following temperature protocol: $16^{\circ} \mathrm{C}$ for $30 \mathrm{~min}, 42^{\circ} \mathrm{C}$ for $30 \mathrm{~min}$ and $85^{\circ} \mathrm{C}$ for $5 \mathrm{~min}$. Samples were then subjected to quantitative PCR using a TaqMan MicroRNA PCR kit (both from Applied Biosystems; Thermo Fisher Scientific, Inc.). The temperature protocol for qPCR was as follows: $50^{\circ} \mathrm{C}$ for $2 \mathrm{~min}, 95^{\circ} \mathrm{C}$ for $10 \mathrm{~min} ; 40$ cycles of denaturation at $95^{\circ} \mathrm{C}$ for $15 \mathrm{sec}$ and annealing/extension at $60^{\circ} \mathrm{C}$ for $60 \mathrm{sec}$. For FGFR1 mRNA detection, total RNA was reverse-transcribed into cDNA using a PrimeScript RT1 reagent kit using the following temperature protocol: $37^{\circ} \mathrm{C}$ for $15 \mathrm{~min}$ and $85^{\circ} \mathrm{C}$ for $5 \mathrm{sec}$. Subsequently, qPCR was conducted using a SYBR Premix Ex Taq ${ }^{\mathrm{TM}}$ (both from Takara Biotechnology Co., Ltd.). The thermocycling conditions were as follows: $5 \mathrm{~min}$ at $95^{\circ} \mathrm{C}$, followed by 40 cycles of $95^{\circ} \mathrm{C}$ for $30 \mathrm{sec}$ and $65^{\circ} \mathrm{C}$ for $45 \mathrm{sec}$. The miR- 652 and FGFR1 mRNA levels were normalized to that of U6 small nuclear RNA and GAPDH, respectively. The primers were designed as follows: miR-652 forward, 5'-ACACTCCAGCTG GGCAACCCTAGGAGAGGGTGC-3' and reverse, 5'-GTG TCGTGGAGTCGGCAATTC-3'; U6 forward, 5'-TGGAACG CTTCACGAATTTGCG-3' and reverse 5'-GGAACGATA CAGAGAAGATTAGC-3'; FGFR1 forward, 5'-CTGGTGACA GAGGACAATG-3' and reverse 5'-AGATCCGGTCAAATA ATGCC-3'; and GAPDH forward, 5'-CCTGGTATGACAACG AATTTG-3' and reverse 5'-CAGTGAGGGTCTCTCTCT TCC-3'. All data were quantified using the $2^{-\Delta \Delta \mathrm{Ca}}$ method (26). RT-qPCR was performed in 3 replicate wells per group and repeated three times.

CCK-8 assay. CCK-8 assay was used to evaluate cell proliferation. After $24 \mathrm{~h}$ incubation, transfected cells were collected and plated into 96 -well plates at a density of $2 \times 10^{3}$ cells/well. A total of $10 \mu \mathrm{l}$ CCK-8 solution (Dojindo Molecular Technologies, Inc.) was added into each well at different time points $(0,1,2$ and 3 days). Transfected cells were then incubated at $37^{\circ} \mathrm{C}$ with $5 \% \mathrm{CO}_{2}$ for an additional $2 \mathrm{~h}$. The absorbance was determined at an optical density of $450 \mathrm{~nm}$ using a microplate reader (Bio-Rad Laboratories, Inc.).

Transwell invasion assay. Transfected cells were collected after $48 \mathrm{~h}$ of incubation and suspended in FBS-free DMEM. A total of $5 \times 10^{4}$ transfected cells resuspended in DMEM without FBS were added to the top chamber of the transwell apparatus pre-coated with Matrigel (both from BD Biosciences) at $37^{\circ} \mathrm{C}$ for $4 \mathrm{~h}$. The bottom chamber was filled with $600 \mu \mathrm{l}$ DMEM supplemented with $10 \%$ FBS. After incubating for $24 \mathrm{~h}$, a cotton swab was used to carefully wipe the cells which had not invaded. The invaded cells were fixed with $95 \%$ methanol at room temperature for $30 \mathrm{~min}$ and stained with $0.5 \%$ crystal violet at room temperature for $30 \mathrm{~min}$. The mean number of cells in five randomly selected fields of view was counted under an inverted light microscope (magnification x200; IX83; Olympus Corporation).

Bioinformatics prediction. TargetScan (Release 7.2, March 2018; www.targetscan.org) and microRNA.org (Release, August 2010; last updated, 2010-11-01; www.microrna. org/microrna/) were used to search for the potential targets of miR-652.

Luciferase reporter assay. The 3'-UTR fragments of FGFR1 with wild-type (wt) or mutant (mut) miR-652 binding site were amplified by Shanghai GenePharma Co.,Ltd. (Shanghai, China) and inserted into the pGL3 vector (Promega Corporation). The chemically generated luciferase reporter plasmids were designated as pGL3-FGFR1-3'-UTR wt and pGL3-FGFR1-3'-UTR mut, respectively. Cells were seeded into 24 -well plates, and co-transfected with miR-652 mimics or miR-NC and pGL3-FGFR1-3'-UTR wt or pGL3-FGFR1-3'-UTR mut using 

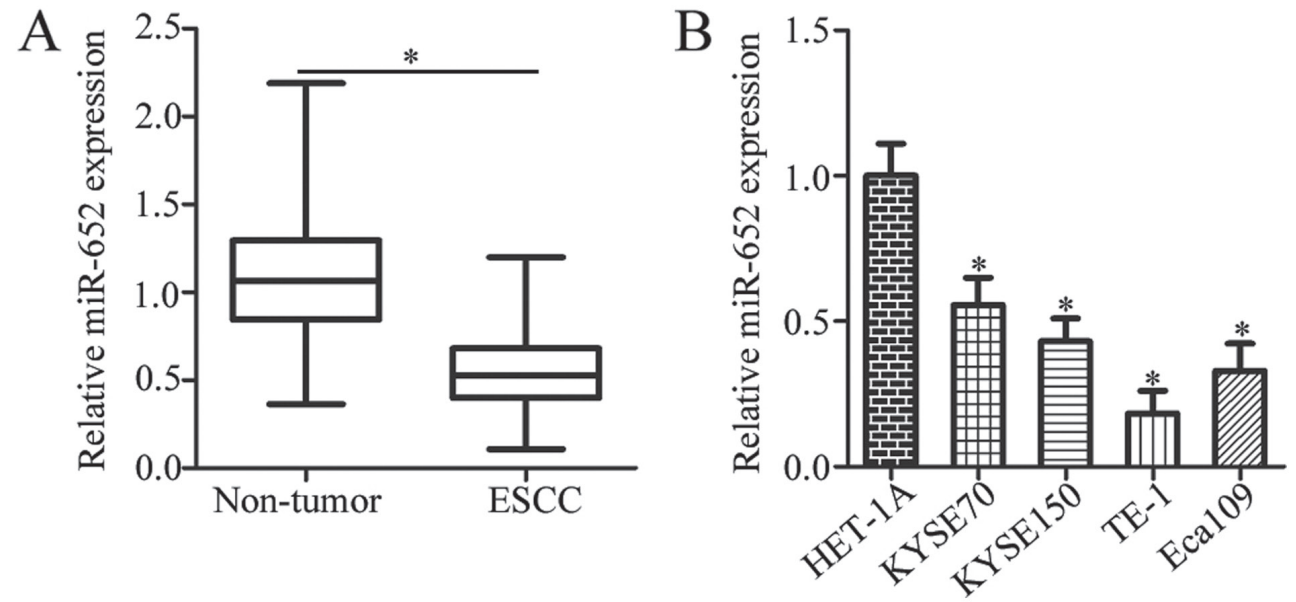

Figure 1. miR-652 is decreased in ESCC tissues and cell lines. (A) Expression of miR-652 was significantly decreased in ESCC tissues compared with matched normal tissue. ${ }^{*} \mathrm{P}<0.05$. (B) Expression of miR-652 in four ESCC cell lines and the normal human esophageal epithelial cell line, HET-1A. ${ }^{*} \mathrm{P}<0.05$ vs. HET-1A. miR-652, microRNA-652; ESCC, esophageal squamous cell carcinoma.

Lipofectamine ${ }^{\circledR} 2000$ reagent according to the protocol specified by the manufacturer. The transfected cells were harvested $48 \mathrm{~h}$ after co-transfection and assayed using a dual-luciferase reporter assay system (Promega Corporation) in accordance with the manufacturer's protocol. Firefly luciferase activity was normalized to that of the Renilla luciferase activity.

Western blot analysis. Total protein was isolated from tissue samples or cells using a Protein Extraction Reagent (Pierce; Thermo Fisher Scientific, Inc.). The concentration of the total protein was detected using a bicinchoninic acid assay kit (Beyotime Institute of Biotechnology). After centrifugation $(14,000 \mathrm{x} \mathrm{g})$ at $4^{\circ} \mathrm{C}$ for $15 \mathrm{~min}$, equal amounts of proteins were subjected to SDS-PAGE (10\% gel) and transferred onto polyvinylidene difluoride membranes (EMD Millipore). After the transfer, the membranes were blocked with 5\% non-fat dried milk in Tris-buffered saline containing $0.1 \%$ Tween-20 (TBST) at room temperature for $1 \mathrm{~h}$, and then incubated with primary antibodies overnight at $4^{\circ} \mathrm{C}$. The primary antibodies used included rabbit anti-human FGFR1 antibody (cat no. ab173305; 1:1,000 dilution; Abcam) and rabbit anti-human GAPDH antibody (cat no. ab181602; 1:1,000 dilution; Abcam). After extensive washing with TBST, the membranes were incubated with horseradish peroxidase-conjugated goat anti-rabbit secondary antibody (cat. no. ab205718; 1:5,000 dilution; Abcam) for $2 \mathrm{~h}$ at room temperature. The protein signals were visualised using an Enhanced Chemiluminescence Plus reagent (GE Healthcare). Quantity One software version 4.62 (Bio-Rad Laboratories, Inc.) was utilized for densitometry.

Statistical analysis. All assays were repeated at least three times. The results are presented as the mean \pm standard deviation and were analyzed with SPSS statistical software (version 11.0; SPSS, Inc.). The differences between groups were examined using a paired Student's t-test, Student's t-test or one-way analysis of variance. A post-hoc Student-Newman-Keuls test was used to test for significance between multiple groups. The correlation between miR-652 and FGFR1 mRNA levels in ESCC tissues was determined using Spearman's rank correla- tion analysis. $\mathrm{P}<0.05$ was considered to indicate a statistically significant difference.

\section{Results}

miR-652 is significantly downregulated in ESCC tissues and cell lines. To illustrate the expression status of miR-652 in ESCC, 37 pairs of ESCC tissues and adjacent non-tumor tissues were collected. miR-652 expression levels were significantly decreased in ESCC tissues compared with that of the adjacent non-tumor tissues (Fig. 1A; P<0.05). Additionally, the expression level of miR-652 was determined in four ESCC cell lines (KYSE70, KYSE150, TE-1 and Eca109) and a normal human esophageal epithelial cell line (HET-1A). The expression levels of miR-652 were lower in the aforementioned ESCC cell lines relative to HET-1A cells (Fig. 1B; $\mathrm{P}<0.05$ ).

miR-652 suppresses the proliferation and invasiveness of ESCC cells. To explore the biological functions of miR-652 in the development of ESCC, miR-652 mimics and miR-NC were chemically synthesized, and then transiently transfected into TE-1 and Eca109 cells which expressed the lowest levels of miR-652 of the four ESCC cell lines. Following transfection, miR-652 was significantly upregulated in TE-1 and Eca109 cells transfected with miR-652 mimics compared with the miR-NC-transfected cells (Fig. 2A; $\mathrm{P}<0.05$ ). The regulatory effect of miR-652 overexpression on ESCC cell proliferation was evaluated by a CCK-8 assay. miR- 652 expression significantly decreased cell proliferation after 2 days compared with the miR-NC-transfected cells in both cell lines (Fig. 2B; $\mathrm{P}<0.05$ ). Additionally, miR-652 upregulation significantly decreased the invasiveness of TE-1 and Eca109 cells (Fig. 2C; $\mathrm{P}<0.05)$. These data thus suggest that miR-652 may serve an inhibitory role in the proliferation and invasion of ESCC cells.

FGFR1 is a direct target gene of miR-652 in ESCC cells. To explore the direct target genes of miR-652 in ESCC cells, online target exploratory programs, TargetScan and microRNA.org, were used to search for the putative targets of miR-652. The 3'-UTR of FGFR1 contains a 7-bp specific 
A
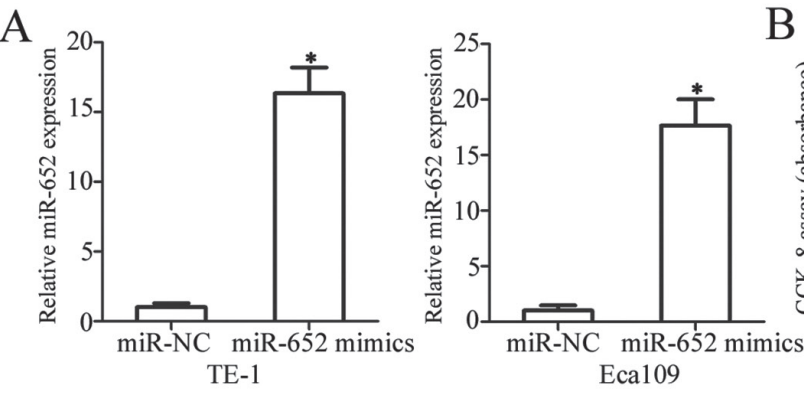

C
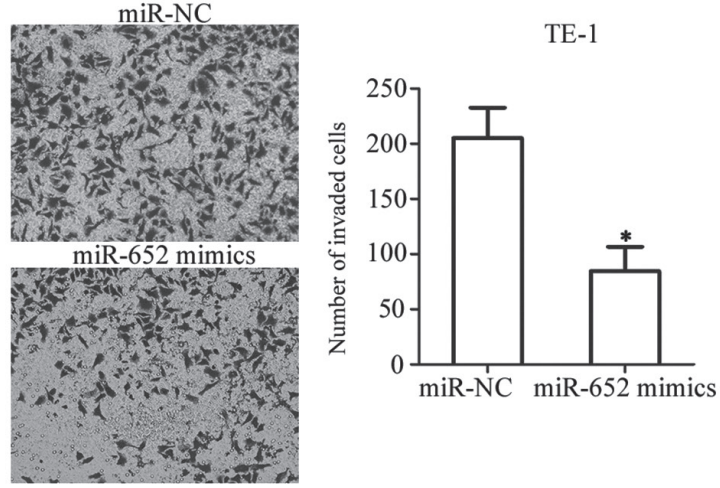

$\mathrm{B}$

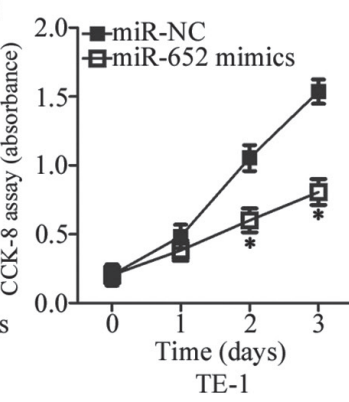

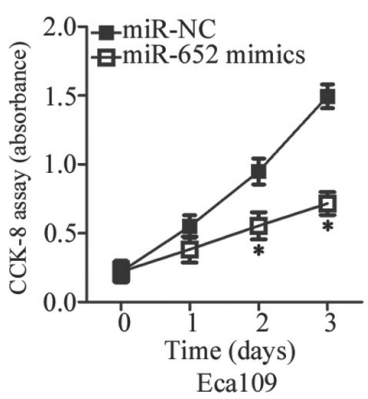

Eca109
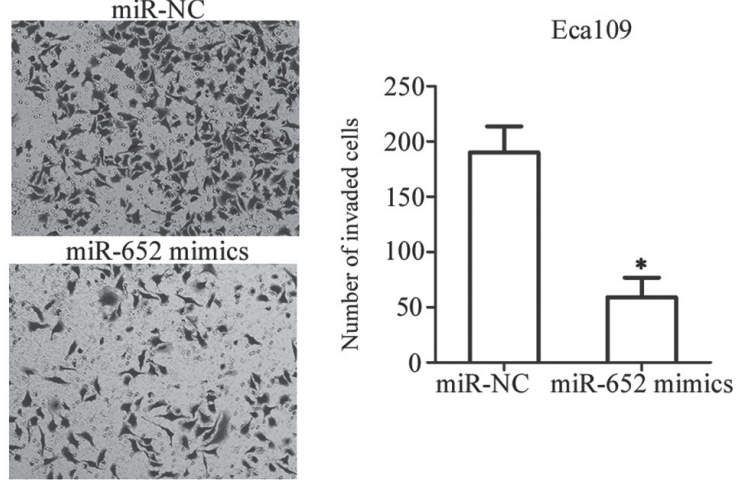

Figure 2. miR-652 inhibits the proliferation and invasion of TE-1 and Eca109 cells. (A) Expression of miR652 was significantly increased in cells transfected with miR-652 mimics. (B) Proliferation was significantly decreased in cells transfected with miR-652 after 2 days. (C) Invasion was significantly decreased in cells transfected with miR-652. "P<0.05 vs. miR-NC. miR-652, microRNA-652; NC, negative control; CCK-8, Cell Counting Kit-8.

complementary sequence which may directly bind miR-652 (Fig. 3A). A total of 398 human genes were predicted as potential targets of miR-652, and FGFR1 was selected for further identification as it was previously reported to be closely associated with ESCC progression (27-31). Luciferase reporter assay was performed to determine whether miR-652 could directly interact with the 3'-UTR of FGFR1. Luciferase activity of the reporter plasmid containing wt FGFR1 3'-UTR was significantly downregulated in TE-1 and Eca109 cells after co-transfection with miR-652 mimics $(\mathrm{P}<0.05)$; however, the luciferase activity of the mut FGFR1 3'-UTR was unaltered (Fig. 3B). To further clarify the regulatory effect of miR-652 on FGFR1, the level of FGFR1 was detected in TE-1 and Eca109 cells transfected with miR-652 mimics or miR-NC. FGFR1 expression was significantly decreased in both ESCC cell lines when transfected with miR-652 mimics compared with the miR-NC-transfected cells at both the mRNA level (Fig. 3C; $\mathrm{P}<0.05$ ) and protein level (Fig. 3D; $\mathrm{P}<0.05$ ). Together, these results suggest that miR-652 may inhibit FGFR1 expression in ESCC cells by directly binding to its 3'-UTR.

FGFR1 expression is increased in ESCC tissues, and its expression is inversely correlated with miR-652 expression. To further explore the association between miR-652 and FGFR1 in ESCC, FGFR1 and miR-652 expression was measured in 37 pairs of ESCC tissues and adjacent non-tumor tissues. The results of RT-qPCR analysis revealed that the expression levels of FGFR1 mRNA were significantly higher in ESCC tissues compared in adjacent non-tumor tissues (Fig. 4A; $\mathrm{P}<0.05$ ). Additionally, western blot analysis verified that the protein expression levels of FGFR1 were increased in ESCC tissues relative to that in adjacent non-tumor tissues
(Fig. 4B; $\mathrm{P}<0.05$ ). Furthermore, Spearman's rank correlation analysis was used to examine the relationship between miR-652 and FGFR1 mRNA levels in ESCC tissues. The expression level of FGFR1 mRNA was inversely correlated with that of miR-652 in ESCC tissues (Fig. 4C; $r=-0.5380$, $\mathrm{P}=0.0006)$.

FGFR1 restoration counteracts the suppressive effects of miR-652 in ESCC cells. Rescue experiments were performed by transfecting FGFR1 overexpression vector lacking 3'-UTR pCMV-FGFR 1 or empty pCMV plasmid into TE-1 and Eca109 cells treated with miR-652 mimics. After transfection, RT-qPCR was firstly performed to determine FGFR1 expression in TE-1 and Eca109 cells after pCMV or pCMV-FGFR1 transfection. FGFR1 mRNA expression significantly increased in the pCMV-FGFR1-transfected TE-1 and Eca109 cells compared with the empty vector group (Fig. 5A, P<0.05). Western blot analysis demonstrated that the FGFR1 protein expression levels were significantly decreased by miR-652 mimics transfection compared with the miR-NC group and recovered in cells co-transfected with miR-652 mimics and pCMV-FGFR1 (Fig. 5B; P<0.05). Restoring FGFR1 expression attenuated the effect of miR-652 mimics on inhibition of proliferation (Fig. 5C; $\mathrm{P}<0.05$ ) and invasion (Fig. 5D; $\mathrm{P}<0.05$ ), respectively. These results suggest that miR-652 inhibits ESCC cell proliferation and invasion, at least partly, by inhibiting FGFR1 expression.

\section{Discussion}

Numerous studies have demonstrated that that a number of miRNAs may be aberrantly expressed in ESCC (32-34). 

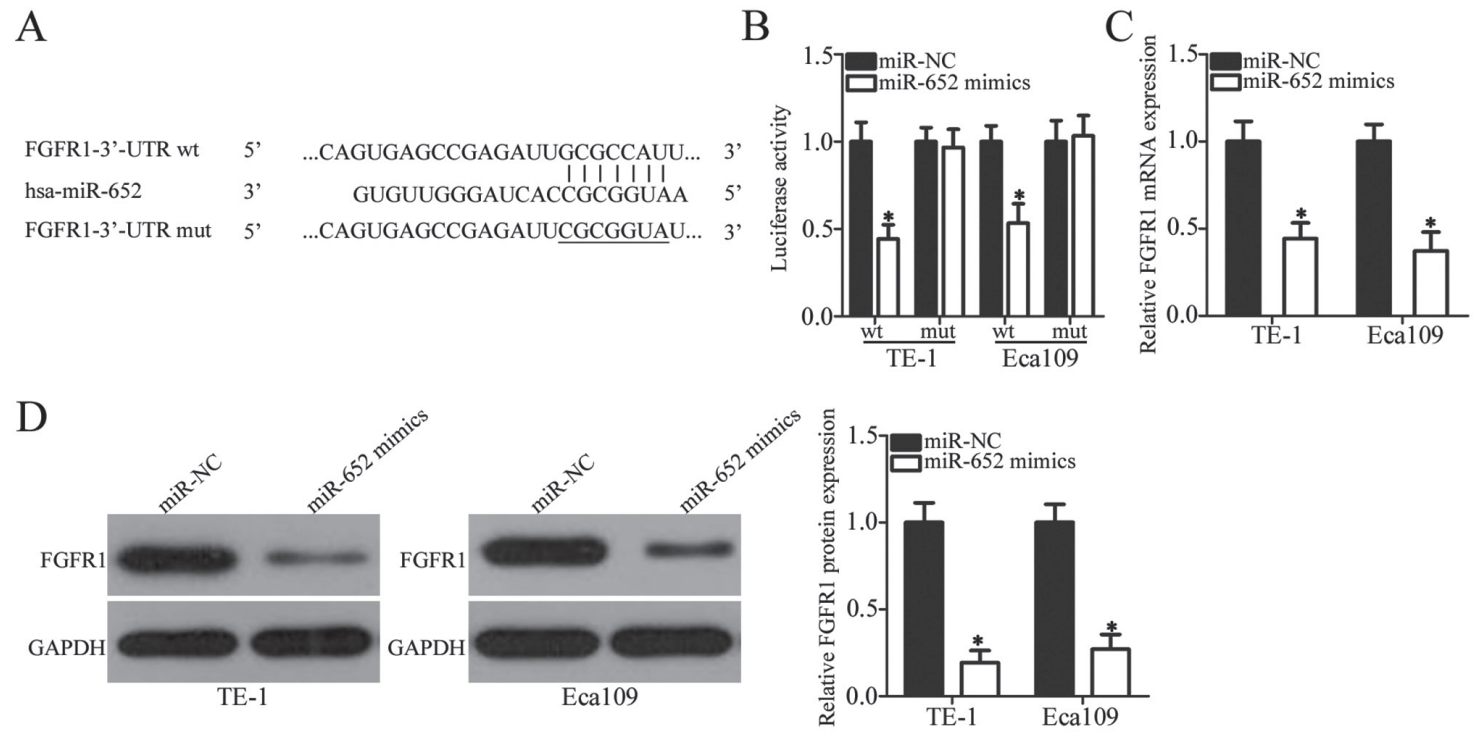

Figure 3. miR-652 directly binds to the 3'-UTR of FGFR1. (A) Wt and mut binding sequences of miR-652 in the 3'-UTR of FGFR1 gene, predicted by bioinformatics analysis. Mut sequences in the 3'-UTR of FGFR1 are underlined. (B) Luciferase reporter assays were performed in TE-1 and Eca109 cells co-transfected with miR-652 mimics or miR-NC, and luciferase reporter plasmids carrying wt or mut miR-652 binding site in the 3'-UTR of FGFR1. (C) mRNA and (D) protein expression levels of FGFR1 in TE-1 and Eca109 cells after transfection with miR-652 mimics or miR-NC. ${ }^{*} \mathrm{P}<0.05$ vs. miR-NC. miR-652, microRNA-652; NC, negative control; 3'-UTR, 3'-untranslated region; FGFR1, fibroblast growth factor 1; wt, wild-type; mut, mutant.

A

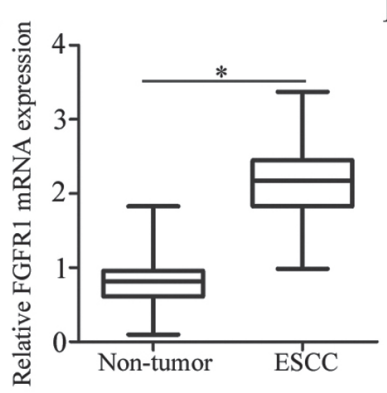

B

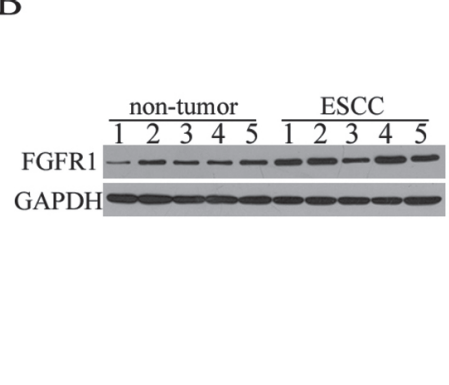

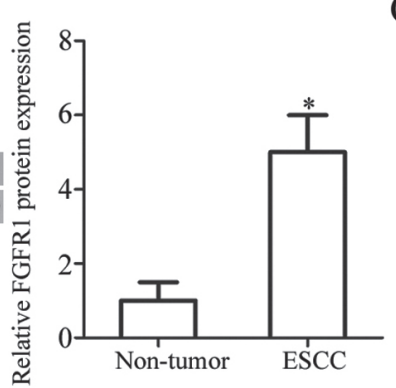

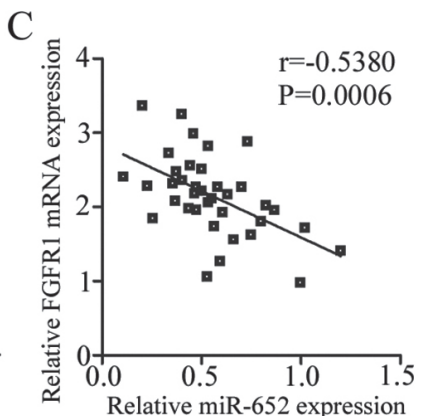

Figure 4. Upregulation of FGFR1 is inversely correlated with miR-652 expression in ESCC tissues. (A) mRNA expression level of FGFR1 in ESCC tissues and adjacent non-tumor tissues. (B) Western blot analysis was used to measure FGFR1 protein expression in ESCC tissues and adjacent non-tumor tissues. $\mathrm{P}<0.05$ vs. adjacent non-tumor tissues. (C) A negative correlation between miR-652 and FGFR1 mRNA levels was identified in ESCC tissues ( $\mathrm{r}=-0.5380$, $\mathrm{P}=0.0006$ ). FGFR1, fibroblast growth factor 1; miR-652, microRNA-652; NC, negative control; ESCC, esophageal squamous cell carcinoma.

Changes in miRNA expression may be associated with ESCC formation and progression, and involved in the regulation of various pathophysiological processes (35-37). Therefore, identification of ESCC-related miRNAs may help elucidate the mechanisms underlying the pathogenesis of ESCC, which may be useful for the development of improved therapeutic approaches for treating patients with ESCC. In the present study, miR-652 was examined in ESCC tissues and cell lines for the first time, to the best of our knowledge. Furthermore, the detailed roles of miR-652 in ESCC progression and the associated underlying mechanisms were examined. These results demonstrated that miR-652 may suppress proliferation and invasion of ESCC cells by targeting FGFR1 directly.

miR-652 is dysregulated in several different types of cancer. For example, miR-652 is upregulated in endometrial cancer tissues and cell lines (22-25). Patients with endometrial cancer with increased expression levels of miR-652 often have reduced overall survival rates and experience earlier recur- rence compared with patients with lower expression levels of miR-652 (22). miR-652 is also overexpressed in non-small cell lung cancer, and associated with lymph node metastasis, Tumor-Node-Metastasis stage and prognosis in non-small cell lung cancer (23). miR-652 expression is often decreased in pediatric acute lymphoblastic leukemia (24) and pancreatic cancer (25). Decreased expression levels of miR-652 are associated with pancreatic cancer stage, lymphatic invasion and metastasis (25). However, to the best of our knowledge, the expression pattern of miR-652 in ESCC has not been reported. In the present study, a total of 37 pairs of ESCC tissues and adjacent non-tumor tissues were collected, and RT-qPCR analysis was performed to detect miR-652 expression levels. The data demonstrated that miR-652 was downregulated in ESCC tissues and cell lines.

miR-652 is implicated as an oncogene or a tumor suppressor gene in the initial stages and progression of human malignancies depending on the characteristic of its target genes. For instance, upregulation of miR-652 promotes endometrial cancer cell 
A

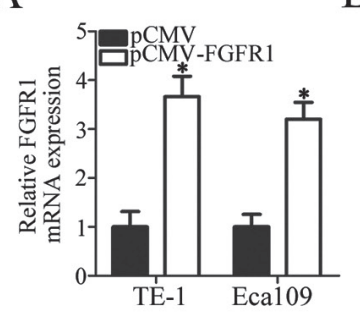

$\mathrm{C}$

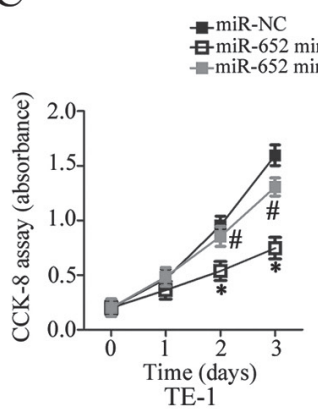

B
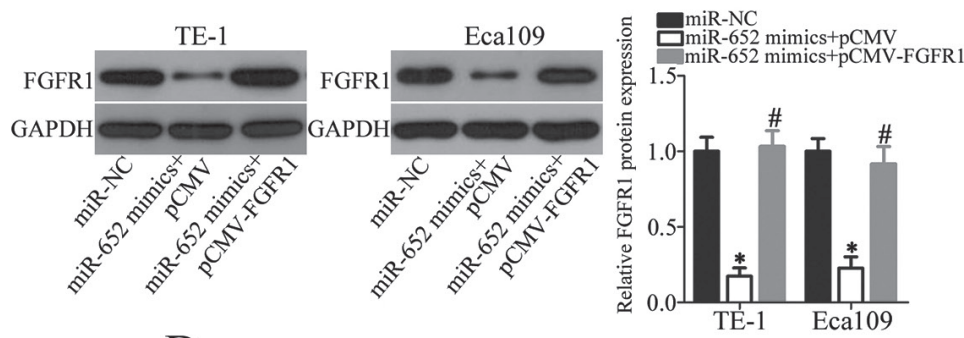

$\mathrm{D}$

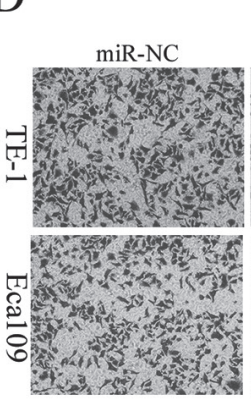

miR-652 mimics+ $\quad$ miR-652 mimics + pCMV

miR-652 mimicst
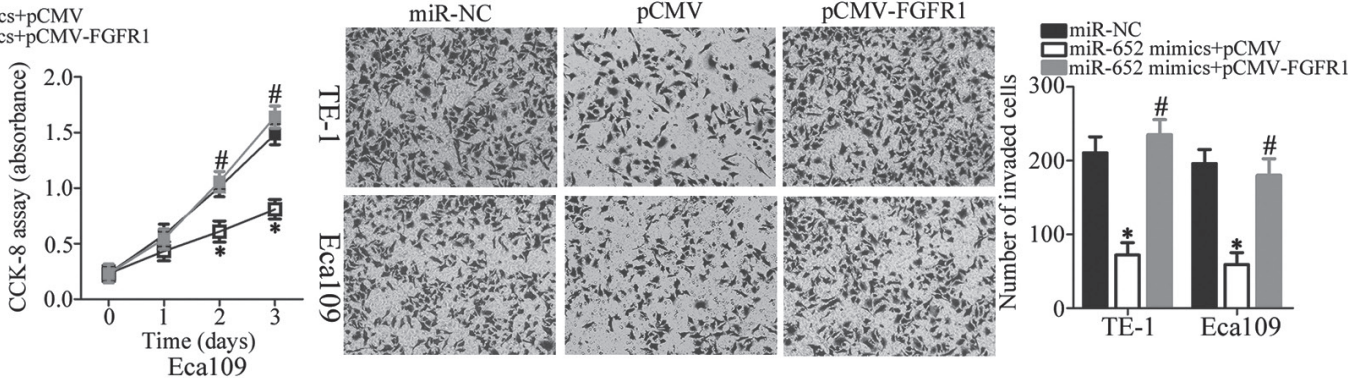

TE-1 Eca109

Figure 5. FGFR1 expression can reverse the phenotypic changes caused by miR-652 overexpression in TE-1 and Eca109 cells. (A) FGFR1 overexpression vector lacking 3'-UTR pCMV-FGFR1 or empty pCMV plasmid was transfected into TE-1 and Eca109 cells. (B) Protein expression level of FGFR1. (C) CCK-8 and (D) transwell invasion assays were used to evaluate the proliferation and invasion of transfected TE-1 and Eca109 cells respectively. $\mathrm{P}<0.05$ vs. miR-NC; ${ }^{\#} \mathrm{P}<0.05$ vs. miR-652 mimics + pCMV. FGFR1, fibroblast growth factor 1; miR-652, microRNA-652; NC, negative control; CCK-8, Cell Counting Kit-8.

growth and metastasis in vitro and in vivo (22). Similarly, in non-small cell lung cancer, miR-652 overexpression increases cell proliferation and motility, and suppresses cell apoptosis in vitro (23). However, miR-652 transfection inhibits the acidity-induced epithelial-mesenchymal transition of pancreatic cancer cells in vitro (25). In pediatric acute lymphoblastic leukemia, upregulation of miR-652 improves the sensitivity to vincristine and cytarabine and induces apoptosis in vitro and in vivo (24). To the best of our knowledge, the biological functions of miR-652 in ESCC cells were not previously studied. In the present study, functional assays of cell behaviors associated with cancer showed that upregulation of miR-652 decreased ESCC cell proliferation and invasion in vitro.

Several genes, including nuclear receptor ROR- $\alpha$ in endometrial cancer (22), lethal (2) giant larvae in non-small cell lung cancer (23) and zinc finger E-box-binding homeobox 1 in pancreatic cancer (25), have been identified as direct targets of miR-652. In the present study, the molecular mechanisms underlying the tumor-suppressing effects of miR-652 in ESCC cells were explored. FGFR1, a member of the fibroblast growth factor family (38), was demonstrated to be a direct target gene of miR-652 in ESCC cells. Increased expression of FGFR1 is observed in multiple types of human cancer, such as gastric (39), bladder (40), lung (41) and breast (42) cancer. In ESCC, FGFR1 is upregulated in tumor tissues (27). ESCC patients with increased FGFR1 expression exhibit reduced disease-free survival and overall survival rates compared with patients with lower levels of FGFR1 (28-30). FGFR1 plays oncogenic roles in the progression and development of ESCC (31).

In conclusion, the present study demonstrated that the expression of miR-652 was downregulated in ESCC tissues and cell lines. Upregulation of miR-652 attenuated cell proliferation and invasion in ESCC. In addition, FGFR1 was validated as a direct target of miR-652 and the roles of miR-652 in
ESCC cells were primarily mediated by suppressing FGFR1 expression. miR-652 may have multiple target genes; however, only FGFR1 was identified as a direct target of miR-652 in ESCC in the current study. The association between miR-652 expression and the survival of ESCC patients was not investigated. These limitations should be addressed in future studies. Additionally, the ability of miR-652 to alter cell biological behaviors in vivo should be investigated.

\section{Acknowledgements}

Not applicable.

\section{Funding}

The present study was funded by The Fund for Scientific Research Activities Shengjing Hospital of China Medical University (Liaoning, China).

\section{Availability of data and materials}

The datasets used and/or analyzed during the present study are available from the corresponding author on reasonable request.

\section{Authors' contributions}

$\mathrm{JL}$ and $\mathrm{CZ}$ designed the study, and performed the experiments. $\mathrm{JH}$ performed the experiments and the statistical analysis. All authors have read approved the final version of the manuscript.

\section{Ethics approval and consent to participate}

The present study was approved by The Ethics Committee of Shengjing Hospital of China Medical University (Liaoning, 
China), and was performed in accordance with the Declaration of Helsinki and the guidelines of the Ethics Committee of Shengjing Hospital of China Medical University. Written informed consent was obtained from all patients recruited in the present study.

\section{Patient consent for publication}

Not applicable.

\section{Competing interests}

The authors declare that they have no competing interests.

\section{References}

1. Cools-Lartigue J, Spicer J and Ferri LE: Current status of management of malignant disease: Current management of esophageal cancer. J Gastrointest Surg 19: 964-972, 2015.

2. Torre LA, Bray F, Siegel RL, Ferlay J, Lortet-Tieulent J and Jemal A: Global cancer statistics, 2012. CA Cancer J Clin 65: 87-108, 2015.

3. Liu B, Jia Y, Cao Y, Wu S, Jiang H, Sun X, Ma J, Yin X, Mao A and Shang M: Overexpression of phosphoserine aminotransferase 1 (PSAT1) predicts poor prognosis and associates with tumor progression in human esophageal squamous cell carcinoma. Cell Physiol Biochem 39: 395-406, 2016.

4. Enzinger PC and Mayer RJ: Esophageal cancer. N Engl J Med 349: 2241-2252, 2003

5. Liu J, Xie X, Zhou C, Peng S, Rao D and Fu J: Which factors are associated with actual 5 year survival of oesophageal squamous cell carcinoma? Eur J Cardiothorac Surg 41: e7-e11, 2012.

6. Toh Y, Egashira A and Yamamoto M: Epigenetic alterations and their clinical implications in esophageal squamous cell carcinoma. Gen Thorac Cardiovasc Surg 61: 262-269, 2013.

7. Song Y, Li L, Ou Y, Gao Z, Li E, Li X, Zhang W, Wang J, Xu L, Zhou Y, et al: Identification of genomic alterations in oesophageal squamous cell cancer. Nature 509: 91-95, 2014.

8. Calin GA and Croce CM: MicroRNA signatures in human cancers. Nature Rev Cancer 6: 857-866, 2006.

9. Kloosterman WP and Plasterk RH: The diverse functions of microRNAs in animal development and disease. Dev Cell 11: 441-450, 2006.

10. Rupaimoole R and Slack FJ: MicroRNA therapeutics: Towards a new era for the management of cancer and other diseases. Nat Rev Drug Discov 16: 203-222, 2017.

11. Iorio MV and Croce CM: MicroRNA dysregulation in cancer: Diagnostics, monitoring and therapeutics. A comprehensive review. EMBO Mol Med 9: 852, 2017.

12. Mei LL, Qiu YT, Zhang B and Shi ZZ: MicroRNAs in esophageal squamous cell carcinoma: Potential biomarkers and therapeutic targets. Cancer Biomark 19: 1-9, 2017.

13. Askandar Iqbal M, Arora S, Prakasam G, Calin GA and Syed MA: MicroRNA in lung cancer: Role, mechanisms, pathways and therapeutic relevance. Mol Aspects Med 17: 300065-300067, 2018.

14. Yang S, Sun Z, Zhou Q, Wang W, Wang G, Song J, Li Z, Zhang Z, Chang Y, Xia K, et al: MicroRNAs, long noncoding RNAs, and circular RNAs: Potential tumor biomarkers and targets for colorectal cancer. Cancer Manag Res 10: 2249-2257, 2018.

15. Yuan HL, Wang T and Zhang KH: MicroRNAs as potential biomarkers for diagnosis, therapy and prognosis of gastric cancer. OncoTargets Ther 11: 3891-3900, 2018.

16. Sharma N and Baruah MM: The microRNA signatures: Aberrantly expressed miRNAs in prostate cancer. Clin Transl Oncol 21: 126-144, 2018.

17. Li B, Xu WW, Han L, Chan KT, Tsao SW, Lee NPY, Law S, Xu LY, Li EM, Chan KW, et al: MicroRNA-377 suppresses initiation and progression of esophageal cancer by inhibiting CD133 and VEGF. Oncogene 36: 3986-4000, 2017.

18. Lindner K, Eichelmann AK, Matuszcak C, Hussey DJ, Haier J and Hummel R: Complex epigenetic regulation of chemotherapy resistance and biohlogy in esophageal squamous cell carcinoma via microRNAs. Int J Mol Sci 19: E499, 2018.
19. Mayne GC, Hussey DJ and Watson DI: MicroRNAs and esophageal cancer-implications for pathogenesis and therapy. Curr Pharm Des 19: 1211-1226, 2013.

20. Xue L, Nan J, Dong L, Zhang C, Li H, Na R, He H and Wang Y: Upregulated miR-483-5p expression as a prognostic biomarker for esophageal squamous cell carcinoma. Cancer Biomark 19: 193-197, 2017.

21. Islam F, Gopalan V, Law S, Tang JC, Chan KW and Lam AK: MiR-498 in esophageal squamous cell carcinoma: Clinicopathological impacts and functional interactions. Human Pathol 62: 141-151, 2017.

22. Sun X, Dongol S, Qiu C, Xu Y, Sun C, Zhang Z, Yang X, Zhang Q and Kong B: MiR-652 promotes tumor proliferation and metastasis by targeting RORA in endometrial cancer. Mol Cancer Res 16: 1927-1939, 2018.

23. Yang W, Zhou C, Luo M, Shi X, Li Y, Sun Z, Zhou F, Chen Z and $\mathrm{He} \mathrm{J}$ : MiR-652-3p is upregulated in non-small cell lung cancer and promotes proliferation and metastasis by directly targeting Lgl1. Oncotarget 7: 16703-16715, 2016.

24. Jiang Q, Lu X, Huang P, Gao C, Zhao X, Xing T, Li G, Bao S and Zheng H: Expression of miR-652-3p and effect on apoptosis and drug sensitivity in pediatric acute lymphoblastic leukemia. BioMed Res Int 5: 5724686, 2018.

25. Deng S, Li X, Niu Y, Zhu S, Jin Y, Deng S, Chen J, Liu Y, He C, Yin T, et al: MiR-652 inhibits acidic microenvironment-induced epithelial-mesenchymal transition of pancreatic cancer cells by targeting ZEB1. Oncotarget 6: 39661-39675, 2015.

26. Livak KJ and Schmittgen TD: Analysis of relative gene expression data using real-time quantitative PCR and the 2(-Delta Delta $\mathrm{C}(\mathrm{T})$ ) method. Methods 25: 402-408, 2001.

27. Sugiura K, Ozawa S, Kitagawa Y, Ueda M and Kitajima M: Co-expression of aFGF and FGFR-1 is predictive of a poor prognosis in patients with esophageal squamous cell carcinoma. Oncol Rep 17: 557-564, 2007.

28. Kim HS, Lee SE, Bae YS, Kim DJ, Lee CG, Hur J, Chung H, Park JC, Jung DH, Shin SK, et al: Fibroblast growth factor receptor 1 gene amplification is associated with poor survival in patients with resected esophageal squamous cell carcinoma. Oncotarget 6: 2562-2572, 2015.

29. Song Q, Liu Y, Jiang D, Wang H, Huang J, Xu Y, Sujie A, Zeng $\mathrm{H}, \mathrm{Xu} \mathrm{C}$ and Hou Y: High amplification of FGFR1 gene is a delayed poor prognostic factor in early stage ESCC patients. Oncotarget 8: 74539-74553, 2017.

30. Wang D, Du L, Wang Z, Liu X, Qin Y, Wang Q, Yang Z, Yao Z, Shi M, Shang B, et al: Association of fibroblast growth factor receptor 1 gene amplification with poor survival in patients with esophageal squamous cell carcinoma. Oncotarget 8: 88857-88869, 2017.

31. Chen B, Liu S, Gan L, Wang J, Hu B, Xu H, Tong R, Yang H, Cristina I, Xue J, et al: FGFR1 signaling potentiates tumor growth and predicts poor prognosis in esophageal squamous cell carcinoma patients. Cancer Biol Ther 19: 76-86, 2018.

32. Ma T, Zhao Y, Lu Q, Lu Y, Liu Z, Xue T and Shao Y: MicroRNA-30c functions as a tumor suppressor via targeting SNAI1 in esophageal squamous cell carcinoma. Biomed Pharmacothe 98: 680-686, 2018.

33. Qi B, Wang Y, Chen ZJ, Li XN, Qi Y, Yang Y, Cui GH, Guo HZ, $\mathrm{Li}$ WH and Zhao S: Down-regulation of miR-30a-3p/5p promotes esophageal squamous cell carcinoma cell proliferation by activating the Wnt signaling pathway. World J Gastroenterol 23: 7965-7977, 2017.

34. Cui XB, Peng H, Li RR, Mu JQ, Yang L, Li N, Liu CX, $\mathrm{Hu}$ JM, Li SG, Wei Y, et al: MicroRNA-34a functions as a tumor suppressor by directly targeting oncogenic PLCE1 in Kazakh esophageal squamous cell carcinoma. Oncotarget 8: 92454-92469, 2017.

35. Yang B, Xie R, Wu SN, Gao CC, Yang XZ and Zhou JF: MicroRNA-615-5p targets insulin-like growth factor 2 and exerts tumor-suppressing functions in human esophageal squamous cell carcinoma. Oncol Rep 39: 255-263, 2018.

36. ZuoJ,Zhu K, Wang Y and Yu Z: MicroRNA-34a suppresses invasion and metastatic in esophageal squamous cell carcinoma by regulating CD44. Mol Cell Biochem 443: 139-149, 2018.

37. Gao X, Xie Z, Wang Z, Cheng K, Liang K and Song Z: Overexpression of miR-191 predicts poor prognosis and promotes proliferation and invasion in esophageal squamous cell carcinoma. Yonsei Med J 58: 1101-1110, 2017.

38. Turner CA, Calvo N, Frost DO, Akil H and Watson SJ: The fibroblast growth factor system is downregulated following social defeat. Neurosci Lett 430: 147-150, 2008. 
39. Schäfer MH, Lingohr P, Sträßer A, Lehnen NC, Braun M, Perner S, Höller T, Kristiansen G, Kalff JC and Gütgemann I: Fibroblast growth factor receptor 1 gene amplification in gastric adenocarcinoma. Human Pathol 46: 1488-1495, 2015.

40. Abdul-Maksoud RS, Shalaby SM, Elsayed WS and Elkady S: Fibroblast growth factor receptor 1 and cytokeratin 20 expressions and their relation to prognostic variables in bladder cancer. Gene 591: 320-326, 2016
41. Wang K, Ji W, Yu Y, Li Z, Niu X, Xia W and Lu S: FGFR1-ERK1/2-SOX2 axis promotes cell proliferation, epithelial-mesenchymal transition, and metastasis in FGFR1-amplified lung cancer. Oncogene 27: 5340-5354, 2018.

42. Cheng CL, Thike AA, Tan SY, Chua PJ, Bay BH and Tan PH: Expression of FGFR1 is an independent prognostic factor in triple-negative breast cancer. Breast Cancer Res Treat 151: 99-111, 2015 